\title{
Estudio preliminar sobre la resistencia al impacto de un material multilaminar
}

\author{
M. Laguna $(*)$ y A.J. Vázquez(*)
}

\begin{abstract}
Resumen Dentro del estudio de materiales resistentes a impactos se analizan los resultados obtenidos con un material multilaminar fabricado con múltiples chapas de acero unidas entre sí por un metal no férreo mediante un proceso de inmersión en metal fundido. Los primeros resultados resultan prometedores, sobre todo teniendo en cuenta el bajo coste de las materias primas y del propio proceso de fabricación.

Palabras clave: Resistencia al impacto. Blindaje. Materiales multilaminares.

\section{Preliminary study of the impact resistance of a multilaminated material}

\begin{abstract}
In the field of impact resistance, some preliminary tests were performed with a new multilaminar material produced by dipping several steel sheets in a molten metal to produce a single piece. Some preliminary tests show an enhancement of the impact resistance. These are very promising results because of the low price of the materials and the low process cost.
\end{abstract}

Keywords: Impact resistance. Armour. Multilaminar composites.

\section{INTRODUCCIÓN}

El ámbito de los materiales resistentes al impacto de interés para el Ministerio de la Defensa es muy amplio y cubre desde blindajes pesados hasta otro tipo de blindajes más ligeros para la protección de vehículos, el propio armamento, instalaciones ligeras y personas frente a proyectiles y explosiones (1).

A lo largo de la historia se ha ido produciendo un desarrollo paralelo entre los blindajes para la protección contra las armas en uso y el desarrollo de nuevas armas capaces de destruirlos. En épocas pasadas, se prestó la mayor atención a las corazas, pero en los momentos actuales, dado el desarrollo de nuevos materiales para blindajes y la capacidad destructora de los elementos ofensivos, resulta difícil aclarar de qué lado está la balanza en el equilibrio coraza/penetrador.

No obstante, la búsqueda de soluciones que procuren la modernización de los blindajes actualmen-

(*) ETS de Ingenieros de Armas Navales, Arturo Soria, 287. 28033-Madrid (España).

(**) Centro Nacional de Investigaciones Metalúrgicas, CENIM (CSIC), Avda. de Gregorio del Amo, 8. 28040Madrid (España). te en servicio es, sin duda, uno de los principales objetivos tecnológicos y estratégicos de los países más avanzados, porque permite alargar la vida útil de equipamientos muy costosos.

Por lo que se refiere a los materiales usados en blindajes, en un principio se utilizaron aceros convencionales, cuyas características mecánicas se iban mejorando intrínsecamente, aumentando su espesor o modificando su inclinación (2). El empleo de espesores muy grandes a base de soldadura planteó el problema de ejecución de este proceso, su falta de homogeneidad y, sobre todo, su peso muerto, por lo cual esta solución resultaba inviable en equipos móviles en los que esta característica era clave.

Algunos ensayos con placas sandwich no dieron los resultados esperados. Posteriormente, Le Creusot presentó la placa Schneider, que consiste en dos placas unidas por laminación con posterior temple y revenido. La fragilidad de estos materiales se corrigió adosando en la parte posterior otra plancha de acero más maleable, material que recibe el nombre de compound. No obstante, los resultados seguían sin ser satisfactorios, probablemente a causa de defectos de unión. Posteriormente, en estos blindajes se emplearon aceros al Cr-Ni-Mo. 
En los últimos años, han aparecido otros blindajes de materiales no férreos, aleaciones de aluminio y, más recientemente, han hecho su aparición blindajes a base de capas fabricados con materiales muy diversos. Asimismo, se ha introducido el concepto de defensa activa, basada en la movilidad, la reducción del gálibo y el despliegue de blindajes reactivos (3), cuyo fundamento reside en la destrucción del misil atacante.

Podría pensarse que el diseño de blindajes pasivos carece de interés hoy día, dado que los misiles modernos de alta energía cinética y carga hueca pueden perforarlos fácilmente, pero no es así. El diseño de blindajes pasivos más eficaces $\mathrm{y}$, sobre todo, con menores pesos y menores precios, sigue siendo un objetivo de gran interés, sobre todo cuando se trata de garantizar la protección frente a amenazas menores, que son las más frecuentes.

En el CENIM se ha desarrollado, en los últimos años, un material compuesto (4) que consiste en la unión de varias láminas de acero por inmersión en un baño de metal fundido, zinc o Pb-Sn. Una vez producida la reacción superficial, las diferentes piezas se extraen simultáneamente y se comprimen antes de que el metal de la superficie solidifique. De este modo, se consigue un material compuesto multilaminar que posee propiedades muy interesantes.

El metal de unión entre las chapas de acero, al tener propiedades de resistencia a la corrosión, confiere a este material unas características de vida en servicio que pueden resultar de gran interés para muchas aplicaciones, en particular, las marinas, donde el ambiente de corrosión es especialmente agresivo. Esta resistencia a la corrosión no se refiere sólo a la corrosión cosmética, sino que, precisamente por su estructura multicapa, presenta también una elevada resistencia a la corrosión perforante.

Algunos estudios, actualmente en curso, consistentes en la exposición de probetas en los arsenales de Cádiz, El Ferrol y Cartagena, en inmersión total, en la zona de mareas y en la zona de salpicaduras, parecen confirmar este buen comportamiento de resistencia a la corrosión (5).

Los ensayos realizados sobre resistencia al impacto, obteniéndose $500-100 \mathrm{~kJ}$, a velocidades muy bajas, del orden de $5-8 \mathrm{~m} / \mathrm{s}$, demostraron la eficacia del material en su resistencia al impacto debida a su estructura multilaminar (6 y 7).

Un trabajo llevado a cabo para ensayos de soldadura por puntos (8) demostró la posibilidad de su unión que, por lo mismo, cabe esperar que pudiera hacérse de un modo continuo, por roldana.

A todas estas ventajas, se añaden dos muy importantes: la del bajo coste de los materiales utilizados y la de la sencillez del proceso, que también repercute en el coste final del producto.

\section{OBJETO DEL TRABAJO}

Aunque es evidente que dadas las características de los impactos balísticos, en los que las velocidades son muy elevadas, es previsible disponer de un material que posea unas características estructurales muy diferentes, se consideró que podría tener un cierto interés el ensayo de resistencia al impacto por proyectiles prefragmentados con este material.

\subsection{Material empleado}

El material multilaminar empleado estaba constituido por cinco chapas de acero de $100 \times 100 \times 1$ $\mathrm{mm}$ de espesor obtenido por inmersión en zinc fundido. Dadas las pequeñas dimensiones de las probetas, se dispusieron agrupadas sobre un bastidor en series de cuatro probetas formando un cuadrado de $100 \times 100 \mathrm{~mm}$, según se puede ver en el esquema de la figura 1.

\subsection{Realización de los ensayos de impacto}

Los ensayos de impacto se llevaron a cabo en una cerca de impactos (Fig. 2), en cuyo centro se coloca el proyectil que se va a explosionar. Con fines comparativos, se dispusieron varias probetas de acero sencillas de 2, 4, 6, 8 y $10 \mathrm{~mm}$ de espesor y el material multilaminar objeto de estudio. El proyectil se colocó a $1 \mathrm{~m}$ de altura del suelo. El material a ensayar se colocó también a la misma altura para conseguir el mayor número de impactos.

Los ensayos se llevaron a cabo con dos tipos de elementos penetradores:

a) proyectil prefragmentado $76 / 62$ Oto Melara (9) cargado con esferas de $3 \mathrm{~mm}$ de diámetro $\mathrm{y}$ una masa de $0,24 \mathrm{~g}$ de volframio, que tiene una densidad de $17,6 \mathrm{~g} / \mathrm{cm}^{3}$ (Fig. $3 a$ )

b) proyectil prefragmentado, de iguales características, excepto que tienen forma cúbica de 4 $\mathrm{mm}$ de arista y una masa de $1,04 \mathrm{~g}$ (Fig. $3 b$ )

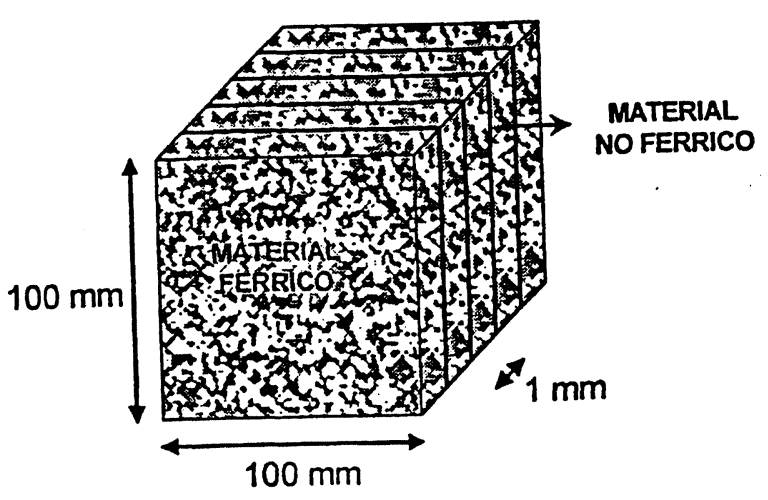

FIG. 1.- Esquema de una chapa multicapa.

FIG. 1.-Scheme of a multilayer plate. 


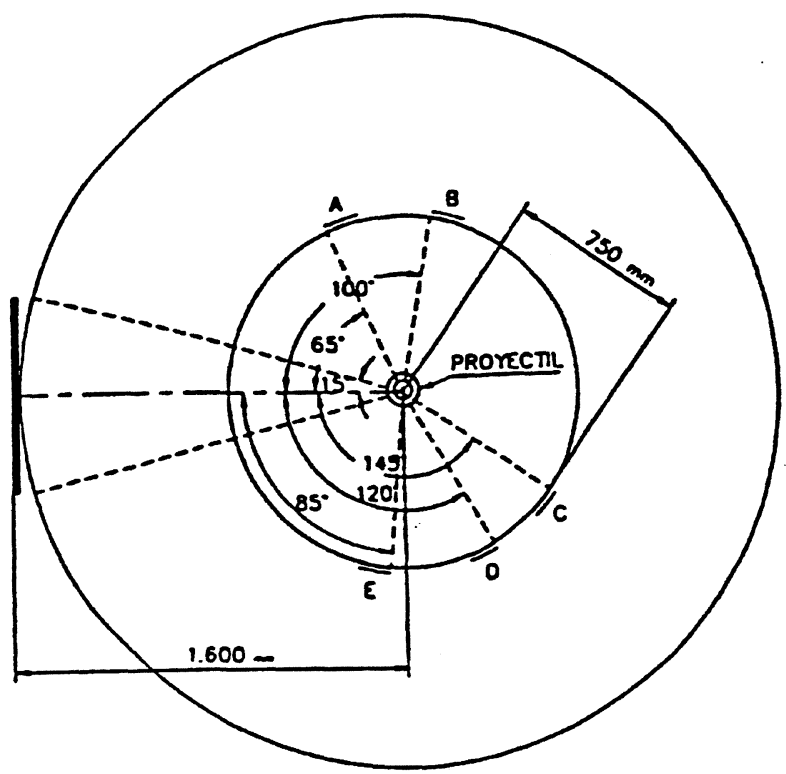

FIG. 2.- Cerca de impactos, disposición del proyectil y de las chapas.

FIG. 2.- Fence of impacts, order of projectile and the plates.

\section{PRESENTACIÓN Y DISCUSIÓN DE LOS RESULTADOS}

Los resultados obtenidos en el ensayo con ambos tipos de proyectiles fueron muy diferentes, por lo que procede analizarlos de modo independiente.

\subsection{Impacto con bolas}

En la tabla I se presentan los resultados obtenidos, donde, como se ve, las perforaciones ocurren sólo en las tres primeras láminas, que se separaron entre sí. Las dos últimas láminas quedaron unidas, aunque también notablemente deformadas.
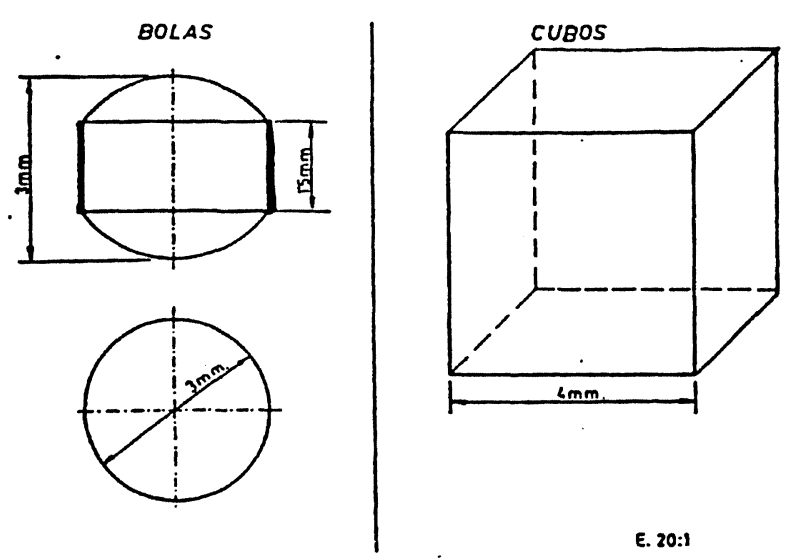

FIG. 3.- Fragmentos: a) esferas, b) cubos.

FIG. 3._Fragments: a) esphere, b) cubes.
En la lámina $4^{\mathrm{a}}$ se puede ver que el número de impactos es superior al de perforaciones de la lámina anterior, como ocurre en los demás casos, porque se consideró que cuando había alguna deformación de la lámina, aunque el proyectil no hubiera perforado la anterior, debía contabilizarse como un impacto. En la figura 4 se ve el aspecto de las distintas láminas después del ensayo.

En la figura 5, que es la habitual para el análisis de este tipo de ensayos, se presenta una gráfica que indica los resultados del impacto en términos porcentuales de perforaciones/impacto vs. número de láminas, magnitud que coincide con el espesor en $\mathrm{mm}$.

\subsection{Impacto con cubos}

El número de impactos fue significativamente menor, pero el poder de penetración fue mucho mayor, ya que, en todos los casos, se produjo la perforación de toda la probeta (Tabla II).

En la figura 6 se ve el aspecto de las perforaciones producidas por los cubos sobre las láminas.

En la figura 7 se presenta la gráfica correspondiente a las perforaciones totales producidas sobre las probetas de acero, de grandes dimensiones y de distinto espesor, tanto en el caso de prefragmentación natural como con bolas y con cubos.

En estos dos últimos casos, que son los que nos ocupan, se puede ver que sólo con espesores de 10 $\mathrm{mm}$ no se produce ninguna perforación. No obstante, en el caso de prefragmentación con bolas, se aprecia que hay una notable disminución del número de perforaciones, que se acerca a cero, cuando el espesor es de $6 \mathrm{~mm}$.

TABLA I.- Impactos y perforaciones con prefragmentados de bolas

TABLE I.- Impact and holes with prefragmented balls

\begin{tabular}{|c|c|c|}
\hline $\begin{array}{c}\text { Muestra } \\
(100 \times 100 \mathrm{~mm})\end{array}$ & $\begin{array}{c}\text { Núm.de } \\
\text { impactos }\end{array}$ & $\begin{array}{c}\text { Núm. de } \\
\text { perforaciones }\end{array}$ \\
\hline $1^{\mathrm{a}}$ lámina & 41 & 38 \\
$2^{\mathrm{a}}$ lámina & 38 & 36 \\
$3^{\mathrm{a}}$ lámina & 36 & 14 \\
$4^{\mathrm{a}}$ lámina & 32 & 0 \\
$5^{\mathrm{a}}$ lámina & $\begin{array}{c}\text { Pegada a la } \\
\text { cuarta }\end{array}$ & 0 \\
\hline
\end{tabular}



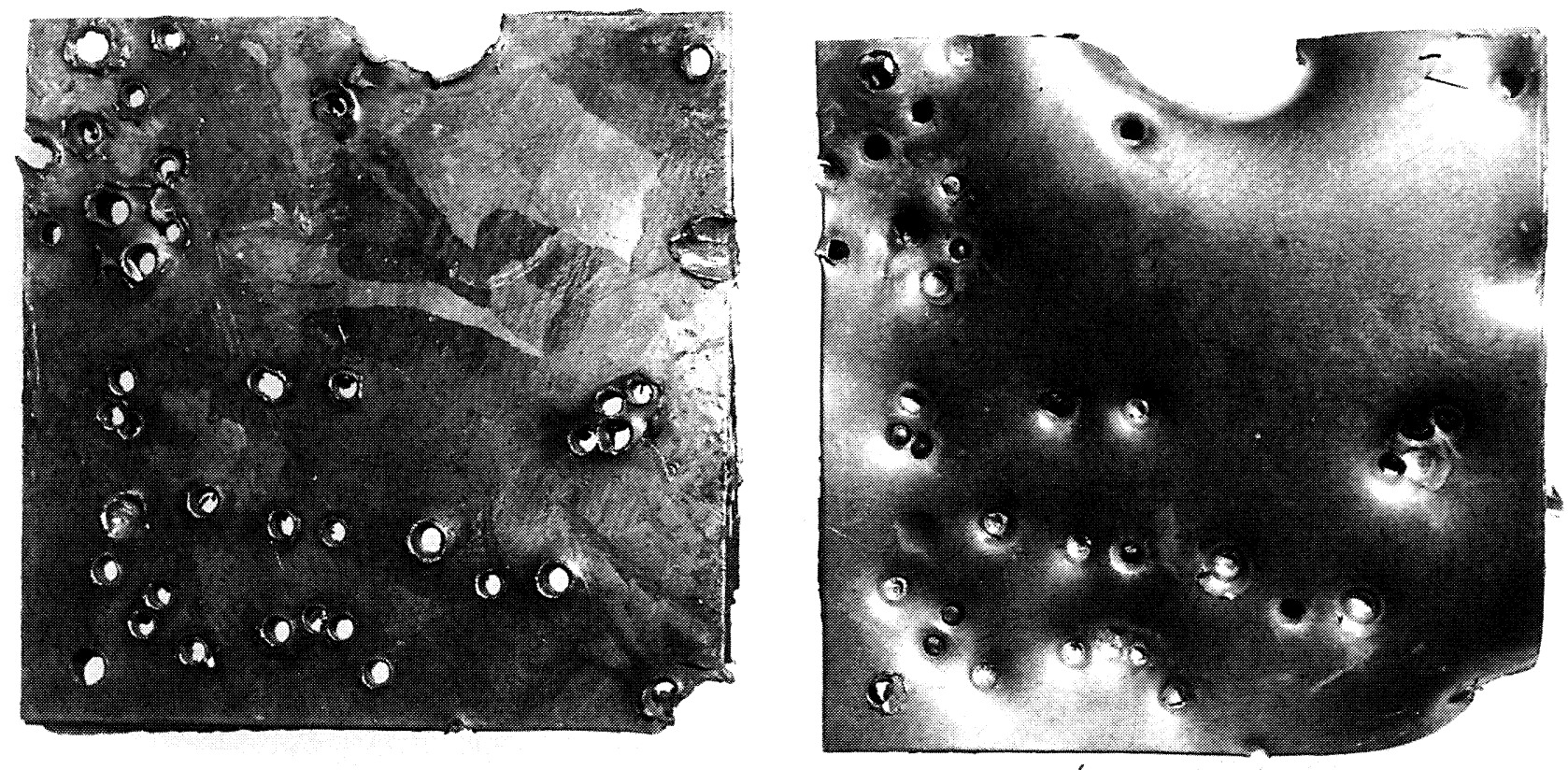

FIG. 4.- Aspecto de las láminas tras el impacto con esferas.

FIG. 4.- Aspect of the sheets after impact with espheres.

\section{CONCLUSIONES}

- Se demuestra que cinco láminas de acero de 1 $\mathrm{mm}$ de grueso, constituyendo un material multilaminar, son mucho más eficaces, como blindaje, frente a proyectiles prefragmentados con forma de bolas, que un blindaje de $6 \mathrm{~mm}$ de acero.

- No se puede obtener ninguna conclusión en el caso de impacto con proyectiles prefragmentados con forma de cubos, porque el espesor de

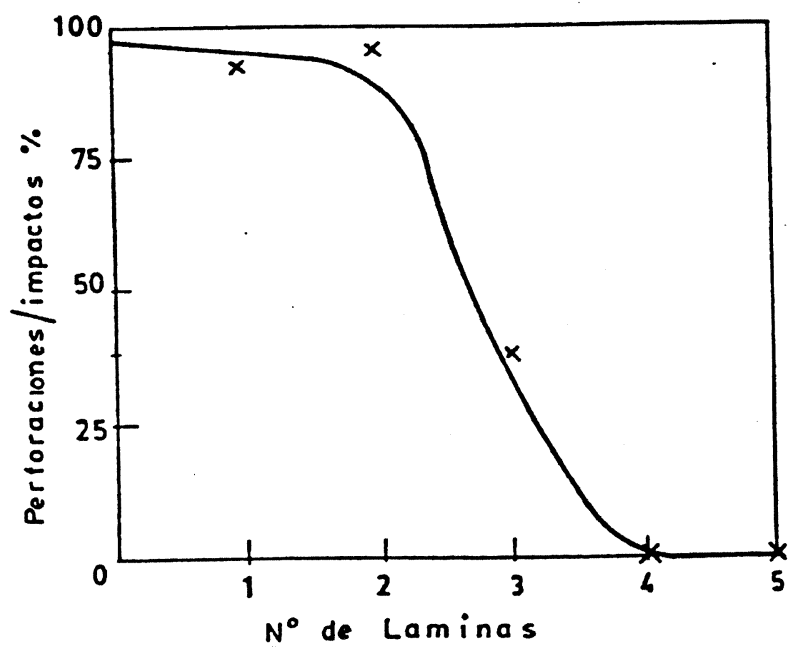

FIG. 5.- Relación perforaciones/impactos vs número de capas.

FIG. 5.- Relation perforations/impacts vs number of layers. la probeta ensayada fue muy pequeño, $5 \mathrm{~mm}$, respecto al necesario en el caso de materiales simples, $10 \mathrm{~mm}$, aunque cabría esperar un resultado igualmente beneficioso que en el caso anterior.

- Es conveniente la continuación de este tipo de ensayos utilizando probetas de mayores dimensiones y modificando el espesor unitario de las láminas para determinar cuál es el número óptimo.

TABLA II.- Impactos y perforaciones con prefragmentados de cubos

TABLE II.- Impacts and holes with prefragmented cubes

\begin{tabular}{|c|c|c|}
\hline $\begin{array}{c}\text { Muestra } \\
(100 \times 100 \mathrm{~mm})\end{array}$ & $\begin{array}{c}\text { Núm.de } \\
\text { impactos }\end{array}$ & $\begin{array}{c}\text { Núm. de } \\
\text { perforaciones }\end{array}$ \\
\hline 1 $^{\mathrm{a}}$ lámina & 9 & 9 \\
$2^{\mathrm{a}}$ lámina & 9 & 9 \\
3 lámina $^{\mathrm{a}}$ lámina & 9 & 9 \\
4 lámina $^{\mathrm{a}}$ lámina & 6 & $6^{*}$ \\
\hline
\end{tabular}

NOTA: *indica que parte de los cubos perforantes de la lámina anterior, por estar cerca del borde, no llegaron a incidir en la siguiente lámina

NOTE: *means that some cubes making holes in previous sheet, near the edge that left the sample, did not impact this sheet. 

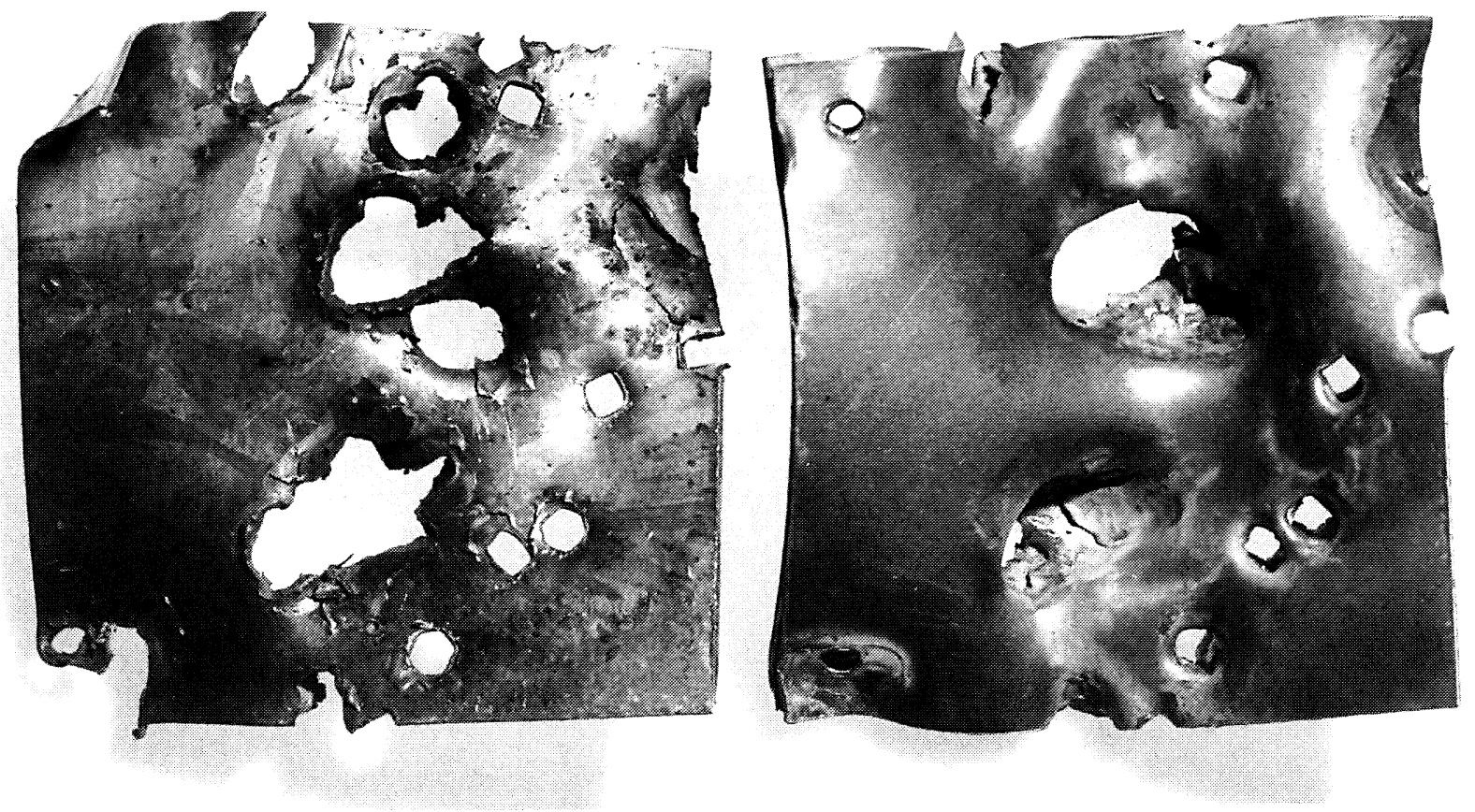

FIG. 6.- Aspecto de las láminas tras el impacto con cubos.

FIG. 6.- Aspect of the sheets after impact with the cubes.

\section{Agradecimiento}

A la CECA por su financiación del proyecto $D e$ velopment of a new laminated composite of steel

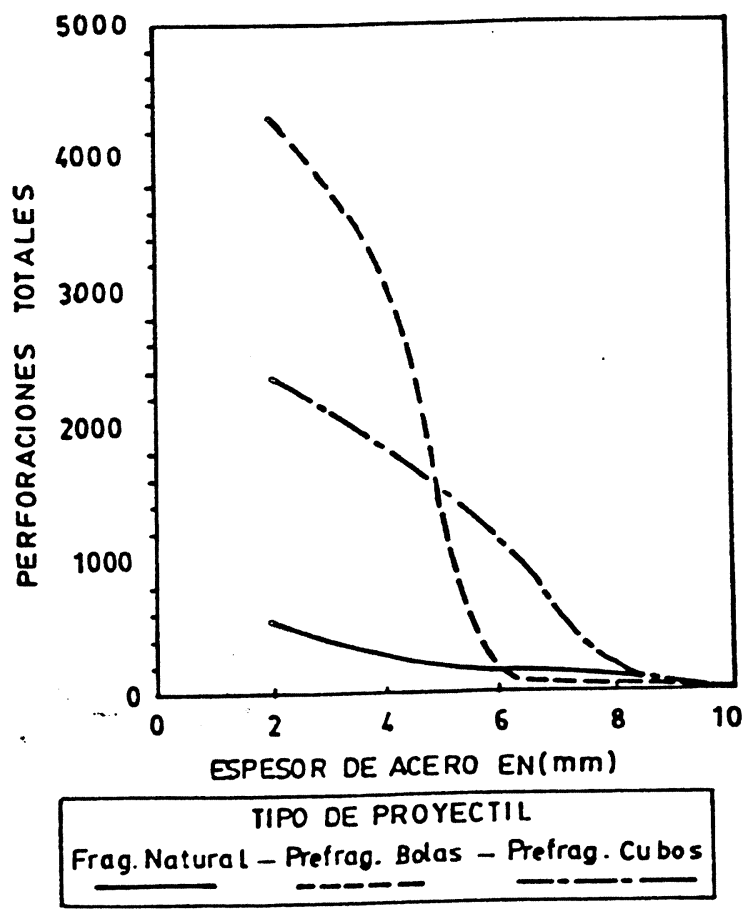

FIG. 7.- Variación en el número de perforaciones en chapas monocapa de diferente espesor.

FIG. 7.- Variation in the number of perforations in the monolayer plates of different thickness. and metallic matrix y a la CICYT por la financiación del proyecto Desarrollo de materiales compuestos multilaminares de matriz metálica. A D.J. Criado, de Expal, S.A., por su asesoramiento en la realización de estos ensayos, y al Cap. J.C. Fernández, del LQCA, por las facilidades puestas a nuestra disposición para realizar estos ensayos.

\section{REFERENCIAS}

(1) Sanchez Galvez, V. Diseño de materiales para blindajes. Materiales Estructurales para la Defensa. ESPOL. Feb. 1995.

(2) Alos Ontiveros, J. Los blindajes homogéneos y su tratamiento térmico. Memorial de Ingeniería de Armamento, núm. 71, 1978.

(3) Anonimo. Sta. Barbara. Blindajes reactivos. Materiales Estructurales para la Defensa. ESPOL. Feb. 1995.

(4) VAzQuez, A.J. y de Damborenea, J.J. Pat. española núm. 8803975. 1990.

(5) LAGUnA, M. Informe sobre resultados de exposición a la corrosión marina de materiales multilaminares. Jornadas CENIM-ETSIAN. Feb., 1995.

(6) VAzQuez, A.J. J. Phys. 3, (4), 1992: 773-781.

(7) VazQuez, A.J. y de Damborenea, J.J. Mater. Design. 14 (3), 1993: 197-201.

(8) Engström, H., Duran, J., Amo, J.M ${ }^{\text {a }}$ y Vazquez, A.J. J. Mater. Sci., 31, (1996), 5443-5449.

(9) LAZARO, A. y CRIADO, J. Seminario sobre diseño y desarrollo de proyectiles PFF fabricados por Explosivos Alaveses, S.A. para la munición del montaje compacto de 76/62 Oto Melara. ETSIAN. Marzo, 1995. 